\title{
Conditions of temperature and time instability occurrence of reverse-biased semiconductor power devices
}

\author{
B. Kojecký ${ }^{\mathrm{a}}$, V. Papež ${ }^{\mathrm{b}, *}$, D. Šámal ${ }^{\mathrm{a}}$

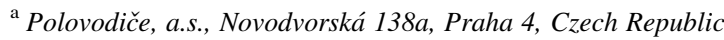 \\ ${ }^{\mathrm{b}}$ Faculty of Electrical Engineering, Department of Electrotechnology, Czech Technical University, Techniká 2, Praha 6, Czech Republic
}

Available online 4 November 2005

\begin{abstract}
An investigation of the boundary states of power semiconductor devices is important with respect to their function reliability. Focus of this article is based on the evaluation of transient temperature increasing of pn-junction under its reverse bias. An influence of positive feedback (heating by some reverse current) is considered on time stability of this reverse current at constant reverse voltage. The temperature is determined which is limiting transiently the reliable function of devices. The problem is solved by two ways. First of them is based on a physical model design describing the heat generation and conduction in semiconductor structure. The second way uses electrical circuit simulation for study of the same structure. Conclusions of both models are applied to collection of experimental data.
\end{abstract}

(C) 2005 Elsevier Ltd. All rights reserved.

Keywords: Silicon power diode; Reverse characteristics; Thermal instability; Reliability

\section{Introduction}

This paper deals with temperature stability of the reverse biased silicon power diodes during their pulse loading. The situation is investigated, when repetitive pulses of a reverse voltage load pn-junction of the diode having defined starting temperature. All these pulses have half-sine shape with amplitude order of $1 \mathrm{kV}$ and corresponding reverse current has its amplitude, belongs to interval $10-50 \mathrm{~mA}$, in dependence on reverse voltage value and starting pn-junction temperature. Massive $\mathrm{Cu}$-electrodes of the device under test are kept at constant temperature and the device is fixed between contact jaws under mounting force about $10 \mathrm{kN}$. The conditions described above are very similar to the ones of a real application.

In power devices, under most conditions, the main part of the losses is generated by forward current. But, some extremal surge forward current can cause that the pn-junction temperature will exceed the maximum working temperature $T_{j \max }$. Under these conditions all producers guarantee a number of following reverse voltage pulses which the device must pass without its destruction.

\footnotetext{
* Corresponding author

E-mail addresses: bkojecky@ polovodice.cz (B. Kojecký), papez@ fel.cvut. cz (V. Papež), dsamal@ polovodice.cz (D. Šámal).
}

0026-2692/\$ - see front matter (C) 2005 Elsevier Ltd. All rights reserved. doi:10.1016/j.mejo.2005.09.030
During each pulse, some power losses are generated in the pn-junction region. It causes heating of silicon structure. Thus, by means of positive feedback, the reverse current is increased more and more. Simultaneously, a part of generated heat is led away by means of massive contact jaws.

After the end of pulse, the temperature of the pn-junction decreases as soon as the accumulated heat is led away. The whole process repeats again with start of a next pulse.

It seems it is reasonable to expect that the system described above will reach its thermo-balance after finite number of pulses. Probability of this prognosis increases with the decrease of starting temperature of the pn-junction. The main feature of the thermo-balance will be characterized by time independent temperature difference between pn-junction and contact jaws.

However, if the common starting temperature both of the pn-junction and the contact jaws increases gradually, the situation can be more complicated. The generated heat will not be led away sufficiently and the pn-junction temperature will increase at any time.

The objective of this is to determine the 'critical' temperature of a concrete power diode (or its pn-junction) when the thermo-balance is not reached for any finite number of pulses.

In practice it means, that the device will be destroyed during a finite time after reverse voltage application.

It is evident that the determination of this temperature is important for the evaluation of reliable behaviour of devices. 
The problem described above is investigated from two points of view. The first of them lies in a design of dynamic model characterizing heat generation and removal in semiconductor structure. This process can be performed by means of differential equations (under simple conditions). The solution of these equations yields a dependence of pn-junction temperature on time and enables formulation of thermal instability condition.

The second way is based on circuit simulation of individual elements of the system. Starting point of this case is the premise that the system can be solved in frequency region. Then, it is possible to create corresponding equations for electric model and to use the Nyquist criteria for their discussion [1].

Results of the theoretical analysis are projected in experimental part of this study. It is represented by real temperature dependences of reverse current for commercial produced diodes. By means of these dependences, the temperature of starting instability is determined.

\section{Models of diode's behaviour under periodical loading of reverse voltage pulses}

\subsection{Description of starting conditions}

Power silicon diode $\left(V_{\mathrm{RRM}}=3800 \mathrm{~V}\right)$ is fixed between contact jaws (double side heating) having high thermal capacity. This equipment guarantees a constant temperature of the device's electrodes during the measurement. The mounting force is $10 \mathrm{kN}$, the pn-junction surface is $21.2 \mathrm{~cm}^{2}$. The thermal resistance $R_{\mathrm{th}}$ (pn-junction to case) is (according the producer) $0.02 \mathrm{KW}^{-1}$.

Starting in time $t=0$, the diode is loaded by half-sine pulses of reverse voltage (length $10 \mathrm{~ms}$, frequency $50 \mathrm{~Hz}$ ). The pn-junction temperature (for $t=0$ is the same as the contact jaws temperature) increases gradually during the time. Generated power losses are very small at first (order of $10 \mathrm{~W})$. That is why the difference between pn-junction temperature and contact jaws temperature is not significant. Under these conditions (small deviation from thermo-balance) the thermal resistance $R_{\mathrm{th}}$ may be considered as a constant and equal to catalogue value presented by the producer.

\subsection{Physical model}

At first, let us consider a situation when one pulse of the reverse voltage is applied with following time out (the both have length equal to $\tau$ ).

Generated power losses during pulse duration are $P_{\mathrm{g}}$. Under the conditions described above, $P_{\mathrm{g}}$ is composed of two parts:

$P_{1}$ is consumed for heat heating the $\mathrm{Si}$-structure

$P_{2}$ is led away out of Si-structure

The balance of the power losses can be described by the following equation.

$P_{\mathrm{g}}=P_{1}+P_{2}$.
Then

$P_{1}=m \cdot c_{\mathrm{s}} \frac{\mathrm{d} \Delta T}{\mathrm{~d} t}$,

where $m$ is the mass of silicon chip, $c_{\mathrm{S}}$ is the specific heat of silicon and $\Delta T$ is the change of temperature related to the initial state.

Similarly for $P_{2}$

$P_{2}=\frac{\Delta T}{R_{\mathrm{th}}}$,

is valid, where $R_{\text {th }}$ is thermal resistance (junction-to case). During the pulse duration, Si chip is heated by actual reverse current. The dependence of reverse current on temperature is approximately exponential in a wide range [2,3]. (A better $P_{\mathrm{g}}$ model would have an $\exp \left(-\mathrm{E}_{\mathrm{g}} / 2 \mathrm{kT}\right)$ dependence for generation current and/or $\exp \left(-\mathrm{E}_{\mathrm{g}} / \mathrm{kT}\right)$ for diffusion current.)

Then

$P_{\mathrm{g}}=P_{0} \cdot \mathrm{e}^{k \Delta T}$,

where $P_{0}$ are the power losses generated in time $t=0$. Then, Eq. (1) can be rewritten in the following form

$P_{0} \cdot \mathrm{e}^{k \Delta T}=m \cdot c_{\mathrm{s}} \cdot \frac{\mathrm{d} \Delta T}{\mathrm{~d} t}+\frac{\Delta T}{R_{\mathrm{th}}}$.

Analytical solution of Eq. (5) is probably complicated.

However, a significant simplification is possible if [4]

(a) $\Delta T$ is only a small deviation; then (using the MacLaurin series),

$P_{\mathrm{g}}=P_{0}\left(1+k \cdot \Delta T+\frac{k^{2} \cdot \Delta T^{2}}{2}\right)$

This step allows precise approximation of the exponential dependence inside a temperature interval of about $40{ }^{\circ} \mathrm{C}$ $\left(R^{2}=0.999\right)$.

(b) half sine shape of pulse may be substituted by rectangular shape; then

$P_{\mathrm{g}}=\frac{U_{0} \cdot I_{0}}{2}\left(1+k \cdot \Delta T+\frac{k^{2} \cdot \Delta T^{2}}{2}\right)$

where $U_{0}, I_{0}$ are amplitudes of half-sine pulses.

Final equation for power losses balance has form

$P_{0}\left(1+k \cdot \Delta T+\frac{k^{2} \cdot \Delta T^{2}}{2}\right)=m \cdot c_{\mathrm{s}} \cdot \frac{\mathrm{d} \Delta T}{\mathrm{~d} t}+\frac{\Delta T}{R_{\mathrm{th}}}$

Putting $\Delta T=y$, and $\Delta T / \mathrm{d} t=y^{\prime}$, Eq. (8) is transformed to the form

$y^{\prime}=a \cdot y^{2}-b \cdot y+c$,

where

$a=\frac{P_{0} k^{2}}{2 m c_{\mathrm{s}}} ; \quad b=\frac{1-k P_{0} R_{\mathrm{th}}}{R_{\mathrm{th}} m c_{\mathrm{s}}} ; \quad c=\frac{P_{0}}{m c_{\mathrm{s}}} ;$ 
Eq. (9) is known as the Ricatti equation. Total solution of this equation requires knowledge at least one particular solution.

So be it $y_{1}$ is some particular solution of the Eq. (9).

Putting

$y_{1}=A=$ const,

then

$y_{1}^{\prime}=0$.

From the physical point of view, $A$ can be a temperature characteristic for equilibrium state.

Substituting $y_{1}, y^{\prime}{ }_{1}$ into Eq. (9), we have (after a simple algebra)

$A_{1,2}=\frac{b \pm \sqrt{b^{2}-4 a c}}{2 a}$.

Because the $A$ must be a real number, then

$D=b^{2}-4 a c \geq 0$,

where $D$ is discriminant of the quadratic Eq. (10).

Substitution of a, b, c into Eq. (10) gives

$b^{2}-4 a c=\frac{1-2 k P_{0} R_{\mathrm{th}}-k^{2} P_{0} R_{\mathrm{th}}}{R_{\mathrm{th}}^{2} m^{2} c_{\mathrm{s}}^{2}} \geq 0$.

or (in this special case)

$k P_{0} R_{\text {th }} \leq \sqrt{2}-1 \leq 0,414$

For $\mathrm{D}=0$ (for one double radix) is

$A=\frac{\sqrt{2}}{k}$.

Solution of Eq. (9) can be looked for in the form

$y=A+z$,

where $z$ is an unknown function. Then, the Eq. (9) is transformed into the Bernoulli equation and (after more extensive modifications) we receive finally

$\Delta T_{1}=A\left\{1+\frac{(2 a A-b)}{[a A-(2 a A-b)] \mathrm{e}^{-(2 a A-b) t}-a A}\right\}$,

where $\Delta T_{1}$ is temperature change of the silicon chip during the length of one pulse.

The second summand in Eq. (16) tends to undetermined value when

$2 a A-b \rightarrow 0$

(from the left). If we consider this expression as an instability condition, then we will have (after substitution of the $a, A, b$ )

$k P_{0} R_{\mathrm{th}}=0.414$.

Now, we can determine starting power losses $P_{0}$ (using a known experimental temperature dependence of the diode reverse current under some defined value of the reverse voltage). This value $P_{0}$ is the one where the instability occurs.
After the termination of the pulse, the accumulated heat is led away and the temperature decreases

$\Delta T_{2}=\Delta T_{1} \mathrm{e}^{-t / R_{\mathrm{th}} m c_{\mathrm{s}}}$.

\subsection{Principle of an electrical model}

The calculation of the inside temperature of electronic device is often based on electrothermal analogy [5]. The system described above can be solved in the frequency region, in other words, the assembled differential equations are modified by means of the Fourier transformation. However, it is more comfortable to create the equations valid for the frequency domain on the basis of an electronic model.

During a diode reverse bias, some power losses are proportional to the corresponding reverse current (which exponentially depends on temperature). An exponential function may be substituted by infinitesimal series. Let us use now the second order multinomial for simple approximation of the exponential function.

The principle of an electrical model is depicted on Fig. 1. The elements R,C represent thermal resistance and thermal capacitance, respectively. The exponential (quadratic) dependence of power losses is performed by a transfer function of the two-port $\mathrm{D}$ (see also Fig. 1). The generator $G$ is a source of signal which represents power losses linked with chip loading. It simulates a positive temperature feedback during one pulse duration.

The electric circuit may be described by equations as follows

$I_{2}=k\left(U_{1}-U_{0}\right)^{2}$,

resp.

$I_{2}=k\left(U_{2}+U_{\mathrm{G}}-U_{0}\right)^{2}$,

$I_{2}=Y U_{2}$,

resp.

$I_{2}=U_{2}\left(\frac{1}{R}+j \omega C\right)$,

$\frac{U_{2}}{R}+j \omega C U_{2}=k\left(U_{2}+U_{\mathrm{G}}+U_{0}\right)^{2}$,

$\frac{U_{2}}{R}+j \omega C U_{2}=I_{2}\left(U_{2}\right)$

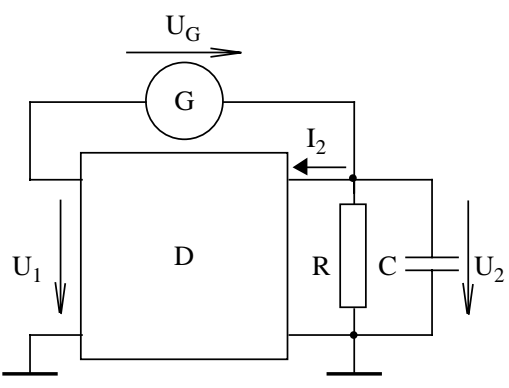

Fig. 1. The principle of an electrical model. 
The symbols $I, U$ correspond to $P, T$, respectively. The term $U_{2}$ is equal to $\Delta T(t) k$, and $I_{2}\left(U_{2}\right)$ is equal $P(\Delta T(t))$.

With respect to this transformation, the Eq. 25 may be rewritten to form

$\frac{\Delta T(t)}{R}+\frac{\mathrm{d} \Delta T(t)}{\mathrm{d} t C}=P(\Delta T(t))$.

It is evident, this equation has the same form as the original Eq. (5) created for thermal quantities.

Now we try to express equivalent transfer values $A, A^{\prime}$ which give a general relations to RMS values of harmonic signals.

$A=\frac{U_{2}}{U_{1}}$,

$A^{\prime}=\frac{U_{2}}{U_{\mathrm{G}}}$.

Because $U_{1}=U_{2}+U_{\mathrm{G}}$,

$A^{\prime}=\frac{A}{(1-A)}$

must be valid. Now, the stability can be solved by means of real circuit substitution by another parametrical linear circuit, which has the transconductance $\mathrm{d} I_{2} / \mathrm{d} U_{1}$. The transconductance depends on parameter $U_{\mathrm{s}}$ which represents DC bias corresponding to operating point of non-linear two-port. Then, if the stability is to be conserved, the denominator of the fraction in Eq. (29) must be different from zero.

According to the Nyquist stability criteria

$\operatorname{Re}(A)<1$,

with respect to

$A=\frac{U_{2}}{U_{1}}=\frac{Z(\omega) \mathrm{d} I_{2}}{\mathrm{~d} U_{1\left(U_{s}\right)}}$.

This condition can be written as follows

$\frac{\operatorname{Re}(Z(\omega)) \mathrm{d} l_{2}}{\mathrm{~d} U_{1\left(U_{\mathrm{s}}\right)}}<1$

If we consider an excitation by a very small signal (linear circuit conditions), the Eq. (32) must be valid for any frequency and any input voltage $U_{\mathrm{s}}$ of the two-port (i.e. for instantaneous temperature).

The impedance $Z(\omega)$ presents frequency dependence. $Z(\omega)$ is the complex function of frequency $\omega$, having maximum value at $\omega=0$. Practically, at ordinary operation, the reciprocal value of time constant $\mathrm{RC}$ is much smaller than the angular frequency of driving signal. Then, the conditions of stability are determined by values of all factors in point $\omega=0$.

For $\omega=0$, i.e. for RMS of all values in periodic steady state it can be written: $\mathrm{Rd} I_{2} / \mathrm{d} U_{1}\left(1\right.$ or for thermal quantities $R_{\text {th }}$ $\mathrm{d} P_{\mathrm{S}} / \mathrm{d} T\left(1\right.$, where $P_{\mathrm{S}}$ means power value of the duration of period $\left(P_{\mathrm{s}}=P_{\text {peak }} / 4\right.$ for sinusoidal courses $)$. The maximum temperature designates the maximum working temperature, when a steady state can be achieved.

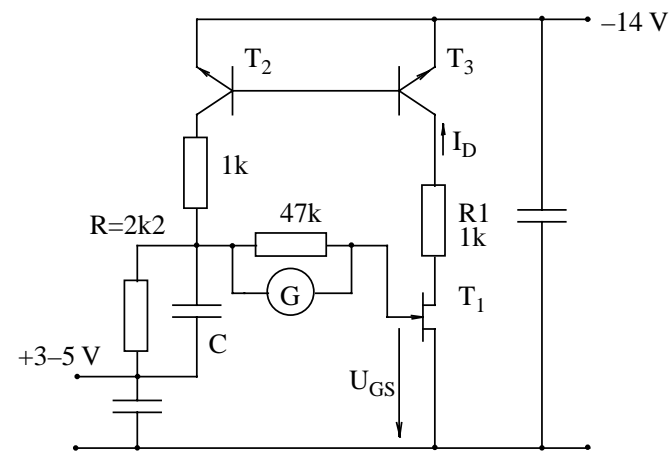

Fig. 2. The scheme of a modelling circuit.

Analogical electronic circuit measurements for validation of analysis are performed. The circuit was realised according to the diagram of Fig. 2.

The non-linear quadratic transfer function (see also Eq. 6) is simulated by transistor JFET $T_{1}$, which embodies the quadratic dependence $I_{\mathrm{D}}$ on $U_{\mathrm{GS}}$. The excitation of transistor $T_{1}$ is realized by generator $G$, which supplies voltage in the form of one-way rectified sinus signal with the frequency of $50 \mathrm{~Hz}$ with controllable amplitude.

Integrator (with controllable time constant) is made of resistor $R$ and a capacitor $C$. The positive feedback is realized by current mirror with transistors $T_{2}$ and $T_{3}$, which feed the current with reverse orientation to integrating circuit $R C$.

\section{Experimental}

The dependence of reverse current $I_{\mathrm{R}}$ on temperature has been investigated at constant value of reverse voltage $V_{\mathrm{R}}$ for a group of power avalanche diodes DA 808-2110-32 (made by Polovodice, a.s., CR). These diodes are designed for max. $V_{\mathrm{R}}$ $=3200 \mathrm{~V}$ and maximum forward current $I_{\mathrm{FAVm}}=2110 \mathrm{~A}$. Maximum value of $I_{\mathrm{R}}$ is $50 \mathrm{~mA}$ at pn-junction temperature of $160^{\circ} \mathrm{C}$. The mass of the silicon chip is $3.00 \mathrm{~g}$, specific heat $c_{\mathrm{s}}$ of silicon is $0.700 \mathrm{~J} \mathrm{Kg}^{-1} \cdot \mathrm{K}^{-1}$, density of silicon is $2.33 \mathrm{~g} \mathrm{~cm}^{-3}$.

The diode under test is fixed between contact jaws (mounting force is about $10 \mathrm{kN}$ ). Before the start of loading, there are the same temperatures of jaws and pn-junction. In the next step, the reverse voltage is applied to the diode (half-sine pulse, $f=50 \mathrm{~Hz}$ ). A corresponding value of reverse current $I_{\mathrm{R}}$ (for max. $V_{\mathrm{R}}$ ) is checked by an oscilloscope. Then, the temperature of jaws is increased and the whole measurement process is repeated.

Measurements described above have been carried out for each diode in a temperature interval $160-210^{\circ} \mathrm{C}$ (limit temperature of equipment). Experimental dependence of $I_{\mathrm{R}}$ vs. temperature jaws is in Fig. 3 (curve a). It seems the exponential approximation is in good accordance with theoretical presumption. For more precision of results interpretation, the total dependence is fitted by two experimental functions.

The course of curve $b$ is derived from the maximum value of $I_{\mathrm{R}}$ approved by producer $\left(I_{\mathrm{R}}=50 \mathrm{~mA}\right.$ at $\left.160{ }^{\circ} \mathrm{C}\right)$. Its shape was estimated with respect to the technological variance of basic 


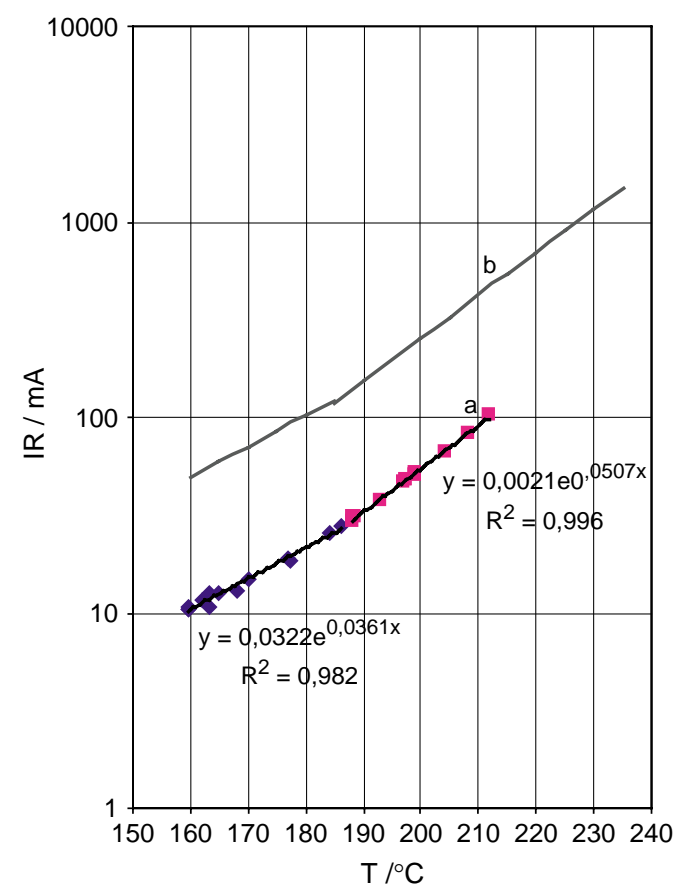

Fig. 3. Experimental (a) and derived (b) dependence of reverse current vs. temperature $\left(V_{\mathrm{R}}=3200 \mathrm{~V}\right)$.

silicon parameters, which influence the value of breakdown voltage (i.e. the value of avalanche multiplication factor). Curve $b$ represents the upper limit of the reverse current value.

\section{Discussion}

Now, we shall compare the results of theoretical models with experimental results.

The fundamental conclusion of the physical model may be expressed by the following formula

$k P_{0} R_{\text {th }} \rightarrow 0.414$

or

$P_{0} \rightarrow \frac{0.414}{k R_{\mathrm{th}}}$ if the system tends to instable state. The value of $R_{\mathrm{th}}$ is given by the producer $\left(R_{\mathrm{th}}=0.02 \mathrm{~kW}^{-1}\right)$. The value of $k$ can be read from Fig. 3 (analytical approximation of the experimental dependence-curve a).

Substituting both $R_{\mathrm{th}}$, and $k$ in Eq. $\left(33^{\prime}\right)$ we have for $P_{0}$

$P_{0} \leq 408 \mathrm{~W}$.

This value corresponds to temperature $T_{\text {in }}$ (instability)

$T_{\text {in }}=230^{\circ} \mathrm{C}$,

where $T_{\text {in }}$ is the temperature for instability start. Note that this temperature concerns a concrete group of diodes under test. In practice, it is necessary to respect some limit of $I_{\mathrm{R}}$ approved by the producer. This limit is caused by technological influences in serial production and it is illustrated by curve b in Fig. 3 . Then, for $P_{0}$

$T_{\text {in }}=202{ }^{\circ} \mathrm{C}$

It is useful to compare this value of $T_{\text {in }}$ with so-called 'intrinsic temperature'. It is defined as a temperature when the density of thermally generated electron-hole pairs is just equal to doping concentration.

If this temperature is reached for a diode under high reverse bias, a microplasma appears in the bulk of the pn-junction. Presence of microplasma increases a probability of disturbances or reliability of devices. The value of intrinsic temperature for diodes DA-808-2110-32 is about $190{ }^{\circ} \mathrm{C}$.

It can be shown analogically that the application of electrical model gives similar results. The typical courses of switching process owing to positive feedback are on Fig. 4. There are shown the courses of $U_{1}=U_{2}+U_{\mathrm{g}}$ and $I_{\mathrm{D}}$ by means of voltage drop a resistor $R_{1}$. The courses of $U_{1}, I_{\mathrm{D}}$ correspond to transformation of the temperature and power losses time dependences. If the gain in positive feedback loop is $<1$ for RMS values (i.e. at a sufficiently low temperature) the electronic circuit is stable. Courses $I_{\mathrm{D}}$ (represent dissipation power of the diode) and $U_{1}$ (represent junction temperature of the diode) are exactly periodical for periodical course of backward voltage.

If the temperature increases, the gain increases too and this is the starting point of instability. The starting region of

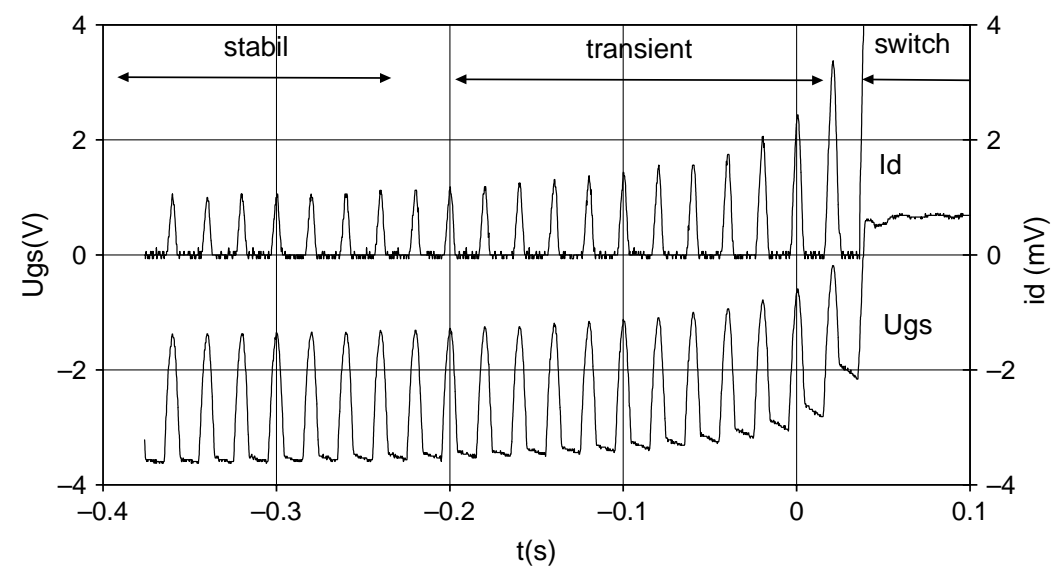

Fig. 4. The typical time courses of switching process. The oscilloscopic picture. 
switching avalanche (start of instability) appears if the gain in positive backward loop is $>1$ for RMS values. Non-periodical component start up of the courses. It means a slow progressive increase of the temperature and dissipation power. It causes a circuit switching or diode breakdown. The transient process is retarded by means of thermal capacity of the diode only. The diode breakdown may be averted only with immediate decrease of the backward voltage.

If the gain of feedback loop is equal to 4 for peak values. Full instability occurs when the gain of the feedback loop is equal to 4 peak values. The electronic circuit switches on and diode break down happens.

$R_{\mathrm{th}} \frac{\mathrm{d} P_{\mathrm{s}}}{\mathrm{d} T}<1 \quad P_{\mathrm{s}}=\frac{P_{\text {peak }}}{4}=\frac{U_{0} I_{0}}{4}$

Back transformation to the circuit symbols gives

$R \frac{\mathrm{d} I_{2}}{\mathrm{~d} U_{1}}<4$

Then, the corresponding values for $T_{\text {in }}$ are

$T_{\text {in }}=254{ }^{\circ} \mathrm{C}$ (for group of tested diodes)

$T_{\text {in }}=222^{\circ} \mathrm{C}$ (for type DA 808-2110-32 generally).

With respect to temperature increase during the pulse, the value $P_{0}$ will be slightly lower. By means of integration of Eq. (2) we will receive (for a short pulse).
$T_{\text {in }}=243{ }^{\circ} \mathrm{C}$ (for group of tested diodes)

$\mathrm{T}_{\mathrm{in}}=212{ }^{\circ} \mathrm{C}$ (for type DA $808-2110-32$ generally)

\section{Conclusions}

The limit conditions of the silicon power diodes function under reverse bias have been determined by means of both physical and electrical circuit models. The temperature of the diode pn-junction was measured and calculated for a case of defined reverse voltage loading with respect to the reliable function of the device. The temperature of about $202-212{ }^{\circ} \mathrm{C}$ was found as a critical value for the start of thermal instability if the device is under ac reverse bias with the frequency of $50 \mathrm{~Hz}$.

\section{References}

[1] A. Angot, Compléments de Mathématiques, Masson et Cie, Paris, 1952.

[2] S.K. Ghandhi, Semiconductor Power Devices, Wiley, New York, 1977, p. 37.

[3] V.Benda, J. Gowar, D.A.Grant, Power Semiconductor Devices-Theory and Applications, Willey, Chichester, 1999, p. 40.

[4] B. Kojecký, V. Papez, D. Sámal, Conditions of Temperature and Time Instability Occurrence of reverse biased Semiconductor Power Devices, ISPS'04 Prague, Sept. 2004, pp. 191-196.

[5] W. Janke, A. Hapka, Simulation of thermal transients in power devices accounting for heat transfer nonlinearities, MicroTherm' 2003 Lodz, pp. 193-197. 